arXiv:1104.5673

\title{
Bounds on Parity Violation In the Cosmological Redshift
}

\author{
Brett Altschul 1 \\ Department of Physics and Astronomy \\ University of South Carolina \\ Columbia, SC 29208 \\ Matthew Mewes \\ Department of Physics and Astronomy \\ Swarthmore College \\ Swarthmore, PA 19081
}

\begin{abstract}
Parity $(\mathrm{P})$ violation in interactions between the spacetime metric and other fields would be a sure sign of new physics. We examine the possibility of $\mathrm{P}$ violation in the cosmological redshift. If right- and left-circularly polarized photons experience the redshift differently, the radiation from distant sources would tend to be depolarized, since the polarization states would accumulate different phases during propagation. The resulting birefringence has an unusual signature - depending on $z^{2}$-quite unlike what is seen in other theories, including those with violation of local boost invariance. The observed broad-spectrum polarization of $\gamma$-ray bursts constrains the fractional difference between the right- and left-handed redshifts at the $6 \times 10^{-37}$ level.
\end{abstract}

\footnotetext{
${ }^{1}$ baltschu@physics.sc.edu
} 


\section{Introduction}

The search for new fundamental physics, especially a quantum theory of gravitation, is an area of fundamental importance. However, finding direct evidence for quantum gravity is expected to be a very difficult problem, because the Planck scale $M_{P}$ is so large compared with the energy scales accessible in particle physics. An alternative way of approaching this problem is to search for evidence of new physics at low energies, by looking for phenomena that cannot occur within the physical theories we already understandthe standard model and general relativity. Such phenomena include violations of charge conservation, Lorentz symmetry, CPT, and the spin-statistics relationship; all these possibilities have been searched for and not seen. However, if any of these exotic phenomena were observed in the laboratory, that would be a discovery of paramount importance and a powerful clue about the nature of new fundamental physics. In this paper, we shall examine another such exotic possibility - a manifestation of parity $(\mathrm{P})$ violation in the cosmological redshift.

There has recently been a substantial amount of work looking at the possibility of $\mathrm{P}$ violation in gravity - a phenomenon that is small in the standard model [1]. Many analyses have focused on Chern-Simons gravity [2], in which the Einstein-Hilbert action is supplemented with a P-violating term that is second order in the curvature. Such a theory could be constrained with measurements of gravitomagnetic effects [3, 4, 5]. Other searches have looked for evidence of gravitational $\mathrm{P}$ violation in conjunction with Lorentz symmetry breaking [6, 7, 8, 9]. In phenomenalistic models (not necessarily described by local field theories) in which right- and left-polarized gravitational waves couple differently, it was also found that there would be P-violating imprints on the cosmic microwave background [10, 11].

It is also possible to study $\mathrm{P}$ violation in phenomena that are not related to the gravitational dynamics but rather to the metrical structure of spacetime. The redshift measures the expansion of the universe, which is the single most important phenomenon in cosmology. We shall examine the possibility that the redshift affects right- and leftcircularly polarized radiation differently. (Such a difference obviously breaks P invariance and potentially other symmetries as well.) If the redshift is an effect purely of geometry, then it should be the same for all electromagnetic radiation; the wavelength is simply stretched out by a constant factor, while waves' local propagation speeds remain uniform. However, the redshift can also be viewed as the result of continual and coherent rescattering of electromagnetic radiation by a time-dependent metric field $g^{\mu \nu}$. If there is $\mathrm{P}$ violation in the photon-metric interaction, it could manifest itself as a difference in redshifts for right- and left-circularly polarized radiation. Like most effects that treat the two polarization states dissimilarly, $\mathrm{P}$ violation in the redshift can lead to birefringence, which may be studied using spectropolarimetry.

There are two ways that a P-violating redshift could depolarize the radiation that

reaches us across cosmological distances. The first way is through the redshift $z=\frac{\lambda_{o}}{\lambda_{e}}-1$ 
(defined in terms of a wave's wavelength at the times of emission and observation) itself. If the left- and right-polarized components of an originally monochromatic wave have different final wavelengths, the wave cannot be purely linearly polarized. However, this turns out to provide a much weaker test of $\mathrm{P}$ invariance than another effect. If the wavelengths of the two polarizations differ during propagation, they will accumulate different phases by the time they are detected. The resulting frequency-dependent difference between the final phases for the two circular components could easily destroy any linear polarization in a broad-spectrum source.

Violation of $\mathrm{P}$ (and $\mathrm{CP}$ ) symmetry in the redshift is a far-reaching effect and would require substantial modification of general relativity. (A non-metric theory of gravitation might be required to accommodate such an effect.) However, we shall not specify the form of the underlying theory responsible for the $\mathrm{P}$ violation. Instead, we shall attempt to describe largely model-independent bounds on the possibility.

It is important that the effect we are considering (like that discussed in [11]) cannot occur in the framework of local field theory, and so the existence of the effect would signal the presence of new physics of a completely novel character. In a local field theory, photon propagation is governed by a gauge-invariant Lagrange density $\mathcal{L}(g, A)$, which is bilinear in the electromagnetic field $A$ and also depends on the (slowly varying) metric $g$. The equivalent problem of a bilinear electromagnetic Lagrange density containing arbitrarily many derivatives was considered in [12], and it was found that no local theory could produce energy-independent differences between the propagation of right- and leftcircularly polarized waves.

Having different phase speeds $c_{ \pm}$for right- and left-polarized waves produces an effect qualitatively similar to $\mathrm{P}$ violation in the redshift, but the signatures of the two phenomena are quite distinct. There are already extremely tight constraints on the kind of birefringence that would be caused by the two polarizations having different phase speeds. Such a variation in the speed of light violates local Lorentz boost invariance, and it can be ruled out at approximately the $10^{-37}$ level [12, 13]. The cited analysis considered only a basis of linear polarization states (although the circular state analysis and results are similar), since an energy-independent difference in phase speeds for the two circular polarization states does not occur in local field theory. However, energy-dependent speed differences between states of circular polarization are possible and have been constrained [14, 15, 16].

\section{Parity Violation in the Redshift}

The effective distance that a photon travels between emission and detection depends on the redshift of the source and the expansion history of the universe. However, the situation simplifies for small redshifts. At lowest order, this distance is then simply $L_{0} \approx z / H_{0}$. If right- and left-circularly polarized waves take different times to cross a constant distance $L_{0}$, this is precisely the same as their having different propagation speeds. Despite the 
presence of $z$ in the formula for $L$, the actual redshift effect is not involved here; instead, we have simply exploited Hubble's Law, which states that $z$ and $L_{0}$ are proportional. The effect we are looking to constrain is different; it entails the two polarizations having different observed redshifts, because their wavelengths expand at rates slightly different from the overall expansion rate of the universe.

The redshift $z(t)$ relates the energy of a given polarization of a wave at different times in its history. For a wave emitted at time $t_{e}$ with energy $k_{e}$ (the same for both polarizations), its energy at a later time $t^{\prime}$ is related by

$$
\frac{k\left(t^{\prime}\right)}{1+z\left(t^{\prime}\right)}=\frac{k_{e}}{1+z\left(t_{e}\right)}
$$

under ordinary circumstances, $z\left(t_{e}\right)$ is simply the redshift $z$ of the source. However, we are interested in what happens if $\mathrm{P}$ violation manifests itself through the right and left polarizations having systematically different $z$ values, $z_{ \pm}$. In that case, the two polarizations' energies will vary differently with time, so that

$$
\delta k\left(t^{\prime}\right) \equiv k_{+}\left(t^{\prime}\right)-k_{-}\left(t^{\prime}\right)=\left[\frac{1+z_{+}\left(t^{\prime}\right)}{1+z_{+}\left(t_{e}\right)}-\frac{1+z_{-}\left(t^{\prime}\right)}{1+z_{-}\left(t_{e}\right)}\right] k_{e} .
$$

In terms of the average redshift $z(t)$ and $\delta z=z_{+}-z_{-}$, we have $z_{ \pm}=\left(1 \pm \frac{1}{2} \frac{\delta z}{z}\right)$. We shall assume $\frac{\delta z}{z}$ is small and constant. (If it is not constant, our final observable will depend on an appropriately weighted average of the $\frac{\delta z}{z}$ values relevant to the propagation period.) It follows that

$$
\delta k \approx\left(\frac{\delta z}{z}\right) \frac{z^{\prime}-z}{(1+z)^{2}} k_{e} \approx\left(\frac{\delta z}{z}\right) \frac{z^{\prime}-z}{(1+z)} k_{o},
$$

where $z^{\prime}=z\left(t^{\prime}\right), z=z\left(t_{e}\right)$ is the redshift of the source, and $k_{o}$ is the energy at the time of observation. The accumulated phase difference between the polarizations at the time of observation is

$$
\delta \phi=\int_{t_{e}}^{t_{o}} d t^{\prime} \delta k\left(t^{\prime}\right)=\left(\frac{\delta z}{z}\right) \frac{k_{o}}{1+z} \int_{0}^{z} d z^{\prime} \frac{z^{\prime}-z}{\left(1+z^{\prime}\right) H\left(z^{\prime}\right)},
$$

in terms of the Hubble expansion parameter $H\left(z^{\prime}\right)$. The plane of polarization for a linearly polarized photon is rotated through an energy-dependent angle $\delta \psi=\frac{1}{2} \delta \phi$.

For our calculations, we shall use the full formula (4) for $\delta \phi$. However, the behavior of $\delta \phi$ for $z \ll 1$ is particularly simple and has a straightforward interpretation. For small $z$, the common phase between the two polarizations is $k_{o} L_{0}$, where $L_{0}$ is the instantaneous distance at the time of emission. The redshift adds to the total travel distance during the propagation time. Since $z \ll 1$, the rate at which extra distance is added to the path is $H_{0}$ times the distance remaining between a traveling wave's position and the Earth. Integrated over the travel time of approximately $L_{0}$, this gives a formula for the distance that is corrected by the expansion of the universe:

$$
L \approx L_{0}+\frac{1}{2} H_{0} L_{0}^{2} \approx L_{0}+\frac{1}{2} z L_{0} .
$$


Since $z$ differs between the two polarization, the phase associated with the added propagation distance $\frac{1}{2} z L_{0}$ differs between the two polarization distances by $-\left(\frac{\delta z}{z}\right) k_{o}$ times $\frac{1}{2} H_{0} L_{0}^{2}$. The phase difference is therefore

$$
\delta \phi \approx-\frac{1}{2}\left(\frac{\delta z}{z}\right) k_{o} \frac{z^{2}}{H_{0}} .
$$

For small $z, \delta \phi$ is proportional not to the distance to the source, but to the distance squared; this is a signature unlike any that would have been seen in theories with a polarization-dependent speed of light. Of course, the characteristic behavior for small $z$ can also be derived from a direct expansion of (4).

\section{Constraints From $\gamma$-Ray Bursts}

The sustained linear polarizations of the $\gamma$-ray bursts GRB 930131, GRB 960924, and GRB 041219a over wide photon energy ranges can be used to rule out a polarization dependence in the redshift quite strongly. Since the rotation of the polarization plane is energy dependent, the existence of a consistent polarization direction across a broad range of energies strongly constrains $\frac{\delta z}{z}$. Such constraints are possible even when the initial polarization direction is unknown.

GRB 930131 and GRB 960924 had measured polarizations over an observed energy range of $31-98 \mathrm{keV}$ of at least $35 \%$ and $50 \%$, respectively [17], and each source had a redshift of at least $z=0.1$. For GRB 041219a, the observed polarization was at least $33 \%$ (at $1 \sigma$ ) in a 100-350 keV energy band [18], and generally consistent results were found in higher-energy bands. The estimated redshift for this burst was $z \approx 0.7$; this estimate was made using a known correlation between the spectral peaks and luminosities of $\gamma$-ray bursts [19].

We used these data to rule out insufficiently small values of $\frac{\delta z}{z}$. The most conservative assumption we could make about the initial polarization of the sources was that the radiation began $100 \%$ linearly polarized, before any birefringence occurred. We have calculated numerically the polarization that would remain to be observed at the Earth, smearing the energy-dependent birefringence over the observed energies. The analysis was performed separately for each source, according to the following procedure. For each one of a large number of possible $\frac{\delta z}{z}$ values, we simulated $10^{4}$ photons. For GRB 930131 and GRB 960924, the photons were distributed across the observational energy range according to a polynomial fit to the measured fluxes. For GRB 041219a, we used a Band model [20] that was known to provide a good fit to the burst's spectrum [21]. We then calculated the total polarization fraction and the rotation of the polarization angle for each sample. We used the Hubble parameter for a $\Lambda$ CDM cosmology with $H_{0}=72 \mathrm{~km} \cdot \mathrm{s}^{-1} \cdot \mathrm{Mpc}^{-1}, \Omega_{m}=0.26$ and $\Omega_{\Lambda}=0.74$. Figure 1 shows the results of these calculations - the surviving degree of polarization (and the rotation of the polarization plane) as a function of $\frac{\delta z}{z}$, including the 


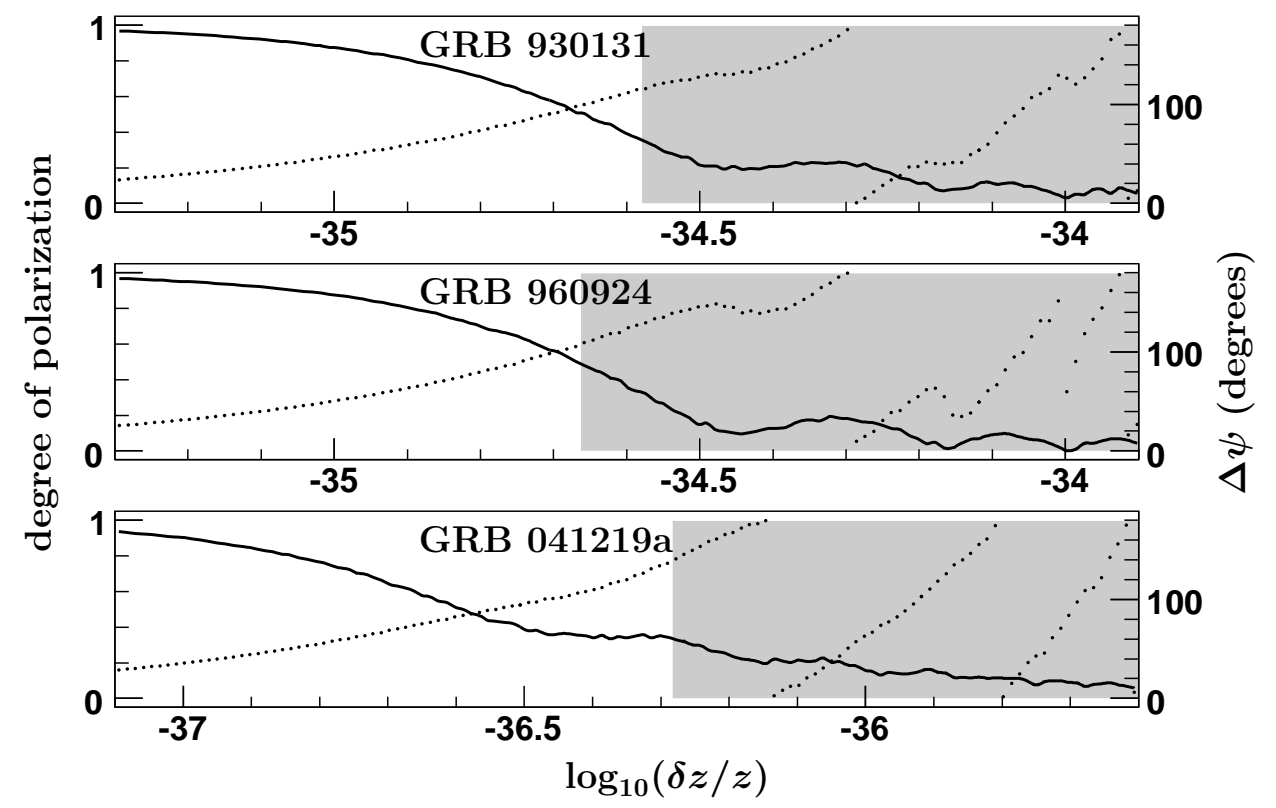

Figure 1: The observed degree of polarization $\Pi$ (solid line) and rotated polarization angle in degrees (dots) for GRB 930131, GRB 960924, and GRB 041219a, assuming the radiation is $100 \%$ linearly polarized at the source, as a function of $\log _{10}(\delta z / z)$. The shaded region is excluded by the bursts' observed levels of polarization.

values of $\frac{\delta z}{z}$ that are ruled out by the respective sources' observed $35 \%, 50 \%$, and $33 \%$ polarizations. The decay in the observed polarization with increasing $\frac{\delta z}{z}$ is pronounced but not strictly monotonic. For small values of $\frac{\delta z}{z}$, the polarizations at different frequencies diverge, but for sufficiently large values of the parameter, the polarizations of different parts of the spectrum may reconverge, when their total rotation angles $\delta \psi$ happen to differ by a multiple of $\pi$.

The characteristic scale of the resulting bounds is

$$
\left|\frac{\delta z}{z}\right| \lesssim \frac{4}{\Delta k L z}
$$

(where $\Delta k$ is the energy range covered by the polarization measurements), indicating bounds at the $10^{-37}-10^{-36}$ level. Taking the polarization and distance parameters given above, the detailed calculations rule out a $\left|\frac{\delta z}{z}\right|$ larger than approximately $3 \times 10^{-35}$ using the GRB 930131 or GRB 960924 data and the somewhat stronger

$$
\left|\frac{\delta z}{z}\right|<6 \times 10^{-37}
$$

with GRB 041219a. The result (8) is our primary bound on P violation in the redshift. 


\section{Other Effects of $\delta z / z$}

It is worthwhile to examine whether another cause of depolarization might also be important experimentally. If the right- and left-circular polarizations from an originally monochromatic wave arrive at the Earth with slightly different wavelengths, the wave train they form cannot remain coherent over its entire length $D$. However, we shall see that the resulting depolarization effect is weaker than the one discussed above by a enormous factor of $L / D$.

An initially linearly polarized monochromatic wave is composed of equal amounts of right- and left-handed radiation, $\vec{E}=\frac{E_{0}}{\sqrt{2}}\left[\hat{\epsilon}_{+} e^{i k_{e}\left(x_{3}-t\right)}+\hat{\epsilon_{-}} e^{i k_{e}\left(x_{3}-t\right)}\right]$. If the circular polarization states are subject to different redshifts $z_{+}$and $z_{-}$, the components will reach the Earth with different wave vectors $k_{ \pm}=k_{e} /\left(1+z_{ \pm}\right)$. If we consider only the difference in frequencies (rather than the phase shifts discussed above), the redshifted wave has the form

$$
\begin{aligned}
\vec{E} & =\frac{E_{0}}{\sqrt{2}}\left[\hat{\epsilon}_{+} e^{i k_{+}\left(x_{3}-t\right)}+\hat{\epsilon_{-}} e^{i k_{-}\left(x_{3}-t\right)}\right] \\
& =E_{0} e^{i k_{o}\left(x_{3}-t\right)}\left\{\hat{x}_{1} \cos \left[\frac{k_{\Delta}}{2}\left(x_{3}-t\right)\right]-\hat{x}_{2} \sin \left[\frac{k_{\Delta}}{2}\left(x_{3}-t\right)\right]\right\} .
\end{aligned}
$$

Here $k_{o}=\left(k_{+}+k_{-}\right) / 2$ and $k_{\Delta}=k_{+}-k_{-}$. At $k_{o}\left(x_{3}-t\right)=0$, the wave maintains its linear polarization along the $x_{1}$-direction. However, ahead of and behind this wave front, the polarization of the wave train is rotated. The wave train as a whole will be strongly polarized only if this rotation is approximately less than $\frac{\pi}{2}$ over the length $D$ of the wave train. More precisely, the Stokes parameters for the wave described by (10), over a wave train of length $D$ centered around $x_{3}=t$ are $\left\langle s^{2}\right\rangle=\left\langle s^{3}\right\rangle=0$, and

$$
\left\langle s^{1}\right\rangle=\frac{\sin \left(k_{\Delta} D / 2\right)}{k_{\Delta} D / 2} I_{0}= \pm \Pi I_{0}
$$

where $I_{0}$ is the intensity, and $\Pi$ is the degree of polarization, which falls off as $\left(k_{\Delta} D\right)^{-1}$.

To determine the wave train length $D$, we must look at how the polarized radiation is emitted. Synchrotron electrons revolve with frequencies $\omega_{B}=\frac{e B}{\gamma m}=\frac{\omega_{c}}{\gamma}$. An orbiting electron radiates up to a cutoff frequency $\omega_{C}=\frac{3}{2} \gamma^{3} \omega_{B} \sin \alpha$, where $\gamma$ is the Lorentz factor of the electron and $\alpha$ is the pitch angle between the direction of $\vec{B}$ and the particle velocity $\vec{v}$. An electron of energy $\gamma m$ emits most of its radiation near $\omega_{C}$, above which frequency the radiated power falls off rapidly. Conversely, radiation with frequency $\omega$ comes mostly from electrons with Lorentz factors $\gamma \sim\left(\omega / \omega_{c}\right)^{1 / 2}$. The radiation from synchrotron electrons is strongly beamed along the direction of their motion. The beam has an angular width of $\Delta \theta \sim \frac{2}{\gamma}$. During each revolution, the charge's direction of motion sweeps through a range of angles $2 \pi \csc \alpha$, and the narrow beam of radiation flashes along the line of sight for a brief time $D_{0} \approx \frac{2}{\gamma \omega_{B}} \csc \alpha=\frac{2 m}{e B} \csc \alpha$. The range of angles into which 
the radiation is emitted depends relatively little on $\omega$ when $\omega \lesssim \omega_{C}$, and the length of the wave train is essentially the pulse duration $D_{0}$ for all relevant emission frequencies.

The observation of a wave with linear polarization $\Pi$ on the Earth requires that $k_{\Delta} D<$

$\frac{2}{\Pi}$. Since $k_{\Delta}=k_{o}(\delta z)$, this produces a bound on $\frac{\delta z}{z}$ that is weaker than (77) by factor of $\sim L / D$. For an optimal source (such as the quasar $3 \mathrm{C} 273$, located at $z=0.158$, where there is clear evidence of polarized synchrotron emission extending from the radio range up into the optical [22]), $\frac{\delta z}{z}$ would need to be at the $10^{-10}$ level or larger for its effects to be detectable in this way.

Inverse Compton (IC) upscattering can increase the energies of polarized synchrotron photons by as much as $4 \gamma^{2}$, but this also tends to decrease the wave train length $D_{0}$. In any case, there are no cosmic electrons energetic enough to produce IC photons for which the depolarization that follows solely from a difference in observed wavelengths would be rapid enough to generate bounds competitive with (7) and (8).

The existence of differing final energies $k_{ \pm}$would also lead to a splitting of the spectral lines seen in redshifted sources. However, direct comparisons of the wavelengths of rightand left-polarized radiation would give constraints many orders of magnitude weaker than (8).

\section{Conclusion}

Just as understanding $\mathrm{P}$ violation is very important to our understanding of particle physics, any $\mathrm{P}$ violation involving couplings to the spacetime metric would be extremely interesting. We have derived a strong bound on any possible $\mathrm{P}$ violation in the cosmological redshift, confirming the basically geometric character of this phenomenon. Although the bound (8) is already very strong, significant improvement will still be possible, as polarization measurements from higher redshift $\gamma$-rays busts become available. Bursts have been observed with redshifts substantially greater than 1 and energies well above 1 $\mathrm{MeV}$, and their true degrees of polarization may be close to $100 \%$. This indicates that the possible improvement could be substantial, although the necessary measurements will naturally be challenging.

\section{References}

[1] Y.-Z. Chu, D. M. Jacobs, Y. Ng, G. D. Starkman, Phys. Rev. D 82, 064022 (2010).

[2] R. Jackiw, S.-Y. Pi, Phys. Rev. D 68, 104012 (2003).

[3] S. Alexander, N. Yunes, Phys. Rev. Lett. 99, 241101 (2007).

[4] T. L. Smith, A. L. Erickcek, R. R. Caldwell, M Kamionkowski, Phys. Rev. D 77, 024015 (2008). 
[5] N. Yunes, D. N. Spergel, Phys. Rev. D 80, 042004 (2009).

[6] J. B. R. Battat, J. F. Chandler, C. W. Stubbs, Phys. Rev. Lett. 99, 241103 (2007).

[7] K.-Y. Chung, S. Chiow, S. Herrmann, S. Chu, H. Müller, Phys. Rev. D 80, 016002 (2009).

[8] Q. G. Bailey, V. A. Kostelecký, Phys. Rev. D 74, 045001 (2006).

[9] H. Panjwani, L. Carbone, C. C. Speake, in Proceedings of the Fifth Meeting on CPT and Lorentz Symmetry, edited by V. A. Kostelecký (World Scientific, Singapore, 2011).

[10] A. Lue, L. Wang, M. Kamionkowski, Phys. Rev. Lett. 83, 1506 (1999).

[11] C. R. Contaldi, J. Magueijo, L. Smolin, Phys. Rev. Lett. 101, 141101 (2008).

[12] V. A. Kostelecký, M. Mewes, Phys. Rev. D 80, 015020 (2009).

[13] V. A. Kostelecký, M. Mewes, Phys. Rev. Lett. 97, 140401 (2006).

[14] S. M. Carroll, G. B. Field, R. Jackiw, Phys. Rev. D 41, 1231 (1990).

[15] R. J. Gleiser, C N. Kozameh, Phys. Rev. D 64, 083007 (2001).

[16] T. Jacobson, S. Liberati, D. Mattingly, F. W. Stecker, Phys. Rev. Lett. 93, 021101 (2004).

[17] D. R. Willis, et al., Astron. and Astrophys. 439, 245 (2005).

[18] S. McGlynn, et al., Astron. and Astrophys. 466, 895 (2007).

[19] D. Yonetoku, et al., Astrophys. J. 609, 935 (2004).

[20] D. Band, et al., Astrophys. J. 413, 281 (1993).

[21] S. McBreen, et al., Astron. and Astrophys. 455, 433 (2006).

[22] H.-J. Röser, K. Meisenheimer, Astron. and Astrophys. 252, 458 (1991). 